\title{
Condiciones de trabajo, estrés y manifestaciones psicosomáticas en médicos de hospitales de la ciudad de México
}

\author{
Working conditions, stress and psychosomatic symptoms in hospitals \\ physicians from Mexico City
}

\author{
Martha Edilia Palacios-Nava', María del Pilar Paz-Román' \\ 1. Departamento de Salud Pública, Facultad de Medicina, Universidad Nacional Autónoma de México - México D.F.
}

Recibido: 20-12-13

Aceptado: 22-04-14

Correspondencia

Martha Edilia Palacios-Nava

Correo electrónico: pnme@unam.mx

Resumen

Introducción. El estrés puede afectar a cualquier grupo ocupacional, aunque algunos presentan una mayor predisposición, tal es el caso de los profesionales de la salud. Objetivo: Evaluar el estrés y su relación con condiciones de trabajo y manifestaciones psicosomáticas en médicos residentes y adscritos. Metodología: Se realizó un estudio transversal con 724 médicos de hospitales de la ciudad de México. Se aplicó un formulario con preguntas acerca de condiciones de trabajo, el inventario de Wolfang, para estrés en profesionales de la salud y el de Kroenke para severidad de manifestaciones psicosomáticas. Resultados: Se encontró una alta consistencia en los inventarios de Wolfang y Kroenke; $61 \%$ de los médicos presentaron estrés moderado y $15 \%$ estrés alto. Se encontraron diferencias significativas de estrés y severidad de los síntomas entre residentes y médicos adscritos; en quienes trabajaban entre 71 y 85 horas por semana; los que realizaban guardias; rotaban turnos y no tenían descansos durante la jornada. Así como asociación entre los niveles de estrés y la severidad de los síntomas. Conclusiones: Las condiciones de trabajo de los residentes mostraron en general, mayor asociación con estrés y sintomatología. Los resultados evidencian la importancia de las condiciones de trabajo en la salud de los médicos.

Med Segur Trab (Internet) 2014; 60 (235) 322-334

Palabras clave: estrés, manifestaciones psicosomáticas, trabajo médico

Abstract

Introduction: Stress can affect any occupational group. Nevertheless the health professionals have a greater predisposition due to the characteristics of their job. Objective: To evaluate the stress and its relation with working conditions and psychosomatic manifestations in physicians. Methodology: A cross sectional study was conducted with 724 residents and medical doctors from Mexico City hospitals. We applied a questionnaire about working conditions, also it was applied the Wolfang stress inventory for health professionals and the Kroenke questionnaire for psychosomatic symptoms. Results: We found high consistency in Wolfing and Kroenke inventories; $61 \%$ of physicians showed moderate stress, $15 \%$ had high stress. We found statistically significant differences in stress and symptoms severity between residents and doctors. Also there were differences among who worked between 71 and 85 hours per week, those who perform guards, rotating shifts and had no breaks during the day. An association between stress levels and severity of symptoms also was found. Conclusions: The working conditions of the residents showed a greater association 
with stress and symptoms. The results showed the importance of working conditions on the health of physicians.

Med Segur Trab (Internet) 2014; 60 (235) 322-334

Key words: Stress, psychosomatic symptoms, physician work 


\section{INTRODUCCIÓN}

\section{Estrés y trabajo médico}

En el desempeño cotidiano del trabajo médico existen múltiples factores de riesgo y exigencias, entre los más importantes se encuentran los factores psicosociales que repercuten en la salud mental de estos profesionistas debido al estrés que generan. Dichos factores, de acuerdo con la Organización Internacional del Trabajo y la Organización Mundial de la Salud, son «interacciones entre el trabajo, su medio ambiente, la satisfacción en el empleo y las condiciones de su organización, por una parte y las capacidades del empleado, sus necesidades, su cultura y su situación personal fuera del trabajo, por la otra; todo lo cual a través de percepciones y experiencias influyen en la salud y el rendimiento" ${ }^{1}$.

Sin embargo, el estrés no siempre tiene una connotación negativa. Éste, tal y como lo definió Hans Selye, es una respuesta específica y «normal» del organismo a una demanda del ambiente externo. En otras palabras, es una respuesta a cualquier situación física, biológica o psicosocial que el sujeto deba afrontar, de tal forma que el estrés no es el estímulo sino la reacción a éste. Por lo tanto, el estímulo estresante puede generar una respuesta positiva (eustress) o una negativa (distress). Esta última depende de la capacidad del individuo para encontrar los recursos necesarios para hacer frente a una situación de emergencia. Cuando esta capacidad es rebasada puede desencadenar patología psicosomática y/o agotamiento funcional ${ }^{2}$.

El estrés producido por el trabajo puede ser definido como respuestas físicas y emocionales dañinas, que aparecen cuando las características del trabajo, no corresponden a la capacidad, recursos y necesidades de los trabajadores ${ }^{3}$. Cuando el estrés laboral es crónico, favorece el decremento de la salud, ejerciendo un efecto desencadenante de síntomas de enfermedad y desgaste. Diversos estudios han mostrado que puede ser origen de diversas patologías físicas, tales como las enfermedades cardiovasculares ${ }^{4}$, mentales, depresión o burnout ${ }^{5,6}$.

El estrés puede afectar a cualquier grupo ocupacional, algunos presentan una mayor predisposición, tal es el caso de los profesionales de la salud cuyas condiciones de trabajo se caracterizan, entre otras cosas, por una situación de sobrecarga, tensión emocional, responsabilidad y supervisión excesiva, jornadas laborales extensas, poca disponibilidad de tiempo para relaciones familiares y sociales, además de otros factores del ambiente físico ${ }^{7}$.

En México, se han llevado a cabo diversos estudios en personal de salud para determinar factores psicosociales, estrés, burnout y algunas condiciones intra y extra laborales asociadas $^{8-11}$. En el caso del estrés la mayoría de estas investigaciones han utilizado instrumentos que no fueron creados específicamente para el trabajo médico, tales como el modelo de Karasek, o la escala de Maslach para determinación del síndrome de burnout, que es una respuesta al estrés crónico y al mismo tiempo una manifestación mayor. ${ }^{8-11}$ Un instrumento diseñado específicamente para la evaluación del estrés en los profesionales de la salud es el inventario de Wolfgang, el cual, a pesar de incluir aspectos muy concretos del trabajo médico, ha sido muy poco utilizado en el ámbito nacional e internacional.

Otro aspecto importante, es que los reportes de investigación publicados en México, en general no reportan las diferencias en las condiciones de trabajo y salud entre médicos adscritos y residentes, pues la mayoría han incluido solo a uno de los grupos o los han comparado principalmente con enfermeras ${ }^{4,6,8-10}$. Además de que la legislación en este país no contempla estas alteraciones como patología profesional de médicos ni enfermeras.

Para contribuir con la evidencia que mostrara la importancia de la problemática y el propósito de encontrar instrumentos adecuados que permitan identificar las características de las condiciones laborales y los efectos en la salud de los médicos, en esta investigación 
se plantearon como objetivos: evaluar las condiciones de trabajo, el estrés específico producido por el trabajo médico y su asociación con manifestaciones psicosomáticas en médicos de hospitales de la ciudad de México y determinar la utilidad tanto del inventario de estrés en el trabajo médico de Wolfang ${ }^{12}$, como del cuestionario de severidad de síntomas de Kroenke ${ }^{13}$.

\section{MÉTODOLOGÍA}

Se llevó a cabo un estudio transversal analítico con 724 médicos de los servicios de urgencias-terapia intensiva, hospitalización y consulta externa de siete hospitales del sector salud en la ciudad de México. La muestra se integró por conveniencia, debido a que se incluyeron la gran mayoría de médicos que laboraban en las áreas mencionadas. Se aplicó un formulario integrado por tres apartados. En el primero se incluyeron preguntas para identificar características socio-demográficas y condiciones de trabajo.

El segundo apartado incluyó el inventario de estrés para profesionales de la salud de Wolfang ${ }^{12}$, el cual consta de 30 preguntas con escala tipo Likert que va de 0 a 4 puntos (nunca, rara vez, ocasionalmente, frecuentemente y muy frecuentemente). Sus variables son reconocimiento personal (9 items), responsabilidad por el cuidado de los pacientes, (7 items), conflictos en el trabajo ( 7 items), e incertidumbre profesional ( 7 items). Una variable general IEPS es el promedio de todos los ítems (se anexa cuestionario). En las publicaciones que se ha reportado el uso de este inventario ${ }^{6,12}$, no se proponen escalas de clasificación ni puntos de corte, para establecer los niveles de estrés, únicamente se ha informado de los promedios de puntos obtenidos por los profesionales de la salud. A pesar de ello, y de que existen otros instrumentos como el de Karasek, en esta investigación se consideró importante validar el inventario de Wolfgang (esta información se reportará en otro texto) y probar su utilidad al relacionarlo con condiciones de trabajo y alteraciones en la salud, debido a que las preguntas que incluye son más específicas del trabajo de estos profesionistas que los de otros instrumentos similares. Para ello, se establecieron cuatro puntos de corte para los niveles de estrés, considerando los puntajes absolutos, y el rango entre estos valores. Así mismo se consideró la pertinencia de utilizar una clasificación similar a la propuesta en el instrumento aplicado para evaluar severidad de síntomas psicosomáticos. Los puntos de corte fueron: de 0 a 30, estrés mínimo o sin estrés; de 31 a 60, estrés moderado; de 61 a 90, estrés alto y de 91 a 120, estrés severo.

Para indagar acerca de manifestaciones psicosomáticas relacionadas con el estrés, se incluyó el instrumento PHQ-15 (Patients health questionnaire), instrumento que ha sido validado y utilizado para evaluar la severidad de los síntomas, incluye 15 preguntas con opciones de respuesta en escala tipo Likert de 0 a 2 (ninguna molestia, molestia ocasional, mucha molestia). Los puntos de corte establecidos son: 0-4 mínimo; 5-9 leve; 10-14 alto y 15-30 severo $^{13}$.

Previo a la investigación se realizó un estudio piloto. La información se analizó con el programa SPSS 19, calculando frecuencias simples, promedio, mediana y desviación estándar en el caso de variables cuantitativas y proporciones para cualitativas, se utilizó Chi cuadrada para analizar las diferencias entre variables socio-demográficas, condiciones de trabajo y su asociación con niveles de estrés y manifestaciones psicosomáticas. Dado que los datos no presentaron una distribución normal, el análisis entre nivel de estrés y severidad de los síntomas se hizo con el coeficiente de correlación de Spearman.

Para la realización del estudio, se siguieron los principios éticos establecidos en la declaración de Helsinki, para la investigación médica en seres humanos 


\section{RESULTADOS}

\section{Características socio-demográficas y condiciones de trabajo}

Participaron 724 médicos, de los cuales $52 \%$ fueron residentes y $48 \%$ médicos adscritos. Del total, el 45\% pertenecía a hospitales de la Secretaría de Salud (SSA), 29\% al Instituto Mexicano del Seguro Social (IMSS) y 26\% al Instituto de Seguridad y Servicios de los Trabajadores del Estado (ISSSTE). El 55\% de la población fueron hombres, la mediana de edad fue de 32 años, $47 \%$ eran solteros y $46 \%$ casados. (Cuadro I)

Cuadro I. Características socio-demográficas de médicos y médicas de siete hospitales de la ciudad de México. México, 2010

\begin{tabular}{|c|c|c|c|c|c|c|c|}
\hline \multirow{2}{*}{$\begin{array}{ll} & \text { Variable } \\
\text { Sexo } & \end{array}$} & \multicolumn{2}{|c|}{$\begin{array}{c}\text { Hombres } \\
\text { No. } \%\end{array}$} & \multicolumn{2}{|c|}{$\begin{array}{c}\text { Mujeres } \\
\text { No. } \%\end{array}$} & \multicolumn{2}{|c|}{$\begin{array}{l}\text { Total } \\
\text { No. } \%\end{array}$} & \multirow[t]{2}{*}{$\mathbf{P}$} \\
\hline & 400 & 55.2 & 324 & 44.8 & 724 & 100.0 & \\
\hline \multicolumn{8}{|l|}{ Categoría en el hospital } \\
\hline Residente & 203 & 50.8 & 174 & 53.7 & 377 & 52.1 & \\
\hline Médico adscrito & 197 & 49.2 & 150 & 46.3 & 347 & 47.9 & \\
\hline Total & 400 & 100.0 & 324 & 100.0 & 724 & 100.0 & 0.428 \\
\hline \multicolumn{8}{|l|}{ Área del sector salud } \\
\hline Secretaría de Salud (3 hospitales) & 186 & 46.5 & 138 & 42.6 & 324 & 44.7 & \\
\hline Instituto de Seguridad y Servicios Sociales de los & & & & & & & \\
\hline Trabajadores del Estado (2 hospitales) & 109 & 27.2 & 82 & 25.3 & 191 & 26.4 & \\
\hline Instituto Mexicano del Seguro Social (2 hospitales & 105 & 26.3 & 104 & 32.1 & 209 & 28.9 & \\
\hline Total & 400 & 100.0 & 324 & 100.0 & 724 & 100.0 & 0.232 \\
\hline \multicolumn{8}{|l|}{ Grupo de edad } \\
\hline $22-27$ & 110 & 27.6 & 90 & 27.7 & 200 & 27.6 & \\
\hline $28-33$ & 108 & 26.9 & 92 & 28.5 & 200 & 27.4 & \\
\hline 34-39 & 33 & 8.2 & 36 & 11.1 & 69 & 9.5 & \\
\hline $40-45$ & 50 & 12.6 & 51 & 15.7 & 101 & 13.9 & \\
\hline $46-70$ & 99 & 24.8 & 55 & 17.0 & 154 & 21.3 & \\
\hline Total & 400 & 100.0 & 324 & 100.0 & 724 & 100.0 & 0.203 \\
\hline \multicolumn{8}{|l|}{ Estado civil } \\
\hline Soltero & 182 & 45.6 & 158 & 48.9 & 340 & 46.9 & \\
\hline Casado o unión libre & 189 & 47.2 & 147 & 45.2 & 336 & 46.4 & \\
\hline Divorciado & 28 & 7.0 & 13 & 4.0 & 41 & 5.7 & \\
\hline Viudo & 1 & .3 & 6 & 1.9 & 7 & 1.0 & \\
\hline Total & 400 & 100.0 & 324 & 100.0 & 724 & 100.0 & 0.044 \\
\hline
\end{tabular}

El promedio de antigüedad fue de 6.7 años, pero la mediana fue de 3, esto debido a que los residentes en general sólo permanecen en los hospitales hasta finalizar su entrenamiento. Respecto a las condiciones de trabajo, estas en general fueron peores para los residentes que para los médicos adscritos. Los primeros tuvieron jornadas de trabajo exhaustivas, 59\% trabajaba entre 70 y 86 hrs, por semana, realización de guardias en el $94 \%$, y aunque ambos grupos reportaron poca libertad para el trabajo, supervisión y carga de trabajo excesivas, estos estuvieron presentes con mayor frecuencia en los residentes. El número de pacientes atendidos fue semejante en ambas categorías, mientras que el tener doble empleo fue mucho mayor en los médicos adscritos (72\%). (Cuadro II) 
Cuadro II. Condiciones de trabajo de residentes y médicos de base de siete hospitales de la ciudad de México. México, 2010

\begin{tabular}{|c|c|c|c|c|c|c|c|}
\hline \multirow{2}{*}{$\begin{array}{c}\text { Variable } \\
\text { Horas trabajadas por seman }\end{array}$} & \multicolumn{2}{|c|}{$\begin{array}{c}\text { Médicos } \\
\text { adscritos } \\
\text { N. }{ }^{\circ} \% \\
\end{array}$} & \multicolumn{2}{|c|}{$\begin{array}{c}\text { Residentes } \\
\text { N. }^{\circ} \%\end{array}$} & \multicolumn{2}{|c|}{$\begin{array}{l}\text { Total } \\
\text { N. }{ }^{\circ} \%\end{array}$} & \multirow[t]{2}{*}{$\mathbf{P}$} \\
\hline & & & & & & & \\
\hline $40-55$ & 225 & 64.8 & 68 & 18.0 & 293 & 40.5 & 0.0001 \\
\hline $56-70$ & 81 & 23.3 & 86 & 22.8 & 167 & 23.0 & \\
\hline $70-86$ & 41 & 11.8 & 223 & 59.2 & 264 & 36.5 & \\
\hline \multicolumn{8}{|l|}{ Realización de guardias } \\
\hline Sí & 120 & 34.6 & 355 & 94.2 & 475 & 65.6 & 0.0001 \\
\hline No & 227 & 65.4 & 22 & 5.8 & 249 & 34.4 & \\
\hline \multicolumn{8}{|l|}{ Rotación de turnos } \\
\hline Sí & 66 & 19.0 & 195 & 51.7 & 261 & 36.0 & 0.0001 \\
\hline No & 281 & 81.0 & 182 & 41.3 & 463 & 64.0 & \\
\hline \multicolumn{8}{|l|}{ N. ${ }^{\circ}$ de pacientes por día } \\
\hline Menos de 15 & 107 & 30.8 & 105 & 27.9 & 212 & 29.3 & 0.135 \\
\hline De 16 a 30 & 223 & 64.3 & 240 & 63.7 & 463 & 64.0 & \\
\hline Más de 31 & 17 & 4.9 & 32 & 8.5 & 49 & 6.8 & \\
\hline \multicolumn{8}{|l|}{ Turno de trabajo } \\
\hline Matutino & 171 & 50.4 & 155 & 41.4 & 326 & 45.7 & 0.001 \\
\hline Vespertino & 88 & 26.0 & 19 & 5.1 & 107 & 15.0 & \\
\hline Nocturno & 20 & 5.9 & 11 & 2.9 & 31 & 4.3 & \\
\hline Jornada acumulada & 22 & 6.5 & 64 & 17.1 & 86 & 12.1 & \\
\hline Otro & 38 & 11.2 & 125 & 33.4 & 163 & 22.9 & \\
\hline \multicolumn{8}{|l|}{ Supervisión excesiva } \\
\hline Nunca & 109 & 31.4 & 51 & 13.6 & 160 & 22.1 & 0.001 \\
\hline A veces & 179 & 51.6 & 175 & 46.5 & 354 & 49.0 & \\
\hline Frecuentemente & 44 & 12.7 & 106 & 28.2 & 150 & 20.7 & \\
\hline Siempre & 15 & 4.3 & 44 & 11.7 & 59 & 8.2 & \\
\hline \multicolumn{8}{|c|}{ Poca libertad para realización del trabajo } \\
\hline Nunca & 155 & 44.7 & 95 & 25.5 & 250 & 34.7 & 0.0001 \\
\hline A veces & 133 & 38.3 & 175 & 46.9 & 308 & 42.8 & \\
\hline Frecuentemente & 47 & 13.5 & 73 & 19.6 & 120 & 16.7 & \\
\hline Siempre & 12 & 3.5 & 30 & 8.0 & 42 & 5.8 & \\
\hline \multicolumn{8}{|l|}{ Carga excesiva de trabajo } \\
\hline Nunca & 28 & 8.1 & 13 & 3.5 & 41 & 5.7 & 0.0001 \\
\hline A veces & 131 & 37.9 & 106 & 28.4 & 237 & 33.0 & \\
\hline Frecuentemente & 132 & 38.2 & 146 & 39.1 & 278 & 38.7 & \\
\hline Siempre & 55 & 15.9 & 108 & 29.0 & 163 & 22.7 & \\
\hline \multicolumn{8}{|l|}{ Tener empleo en otro lugar } \\
\hline Sí & 245 & 71.6 & 54 & 14.4 & 299 & 41.8 & 0.0001 \\
\hline No & 97 & 28.4 & 320 & 85.6 & 417 & 58.2 & \\
\hline
\end{tabular}

\section{Determinación de los niveles de estrés}

Como se mencionó previamente en el apartado de metodología, se aplicó el inventario de estrés para profesionales de la salud, de Wolfang. El alfa de Cronbach de los elementos tipificados en el instrumento fue de 0.915 , lo cual muestra una alta consistencia interna. Con la suma de puntos de cada participante se integró un puntaje global. El promedio obtenido en la población fue de 43.9 con una desviación estándar de 17.98 y mediana de 44 .

De acuerdo con la clasificación establecida, el $61 \%$ de los médicos tenía estrés moderado y el $15 \%$ estrés alto, solo el $1 \%$ presentó estrés severo. (Cuadro III)

\section{Estrés, características socio-demográficas y condiciones de trabajo}

De acuerdo con las características socio-demográficas, los niveles de estrés alto fueron semejantes en ambos sexos, no obstante hubo más casos en el nivel de estrés 
severo en los hombres, $1.6 \%$ contra $0.3 \%$ en mujeres, $\mathrm{p}=0.003$. Respecto a la edad y el estado civil, no hubo diferencias.

Según las condiciones laborales, se encontró diferencia significativa entre hospitales, uno del ISSSTE y otro de la SSA tuvieron porcentajes mayores de estrés en el nivel alto que los del total de la población estudiada, $23 \%$ y $21 \%$ respectivamente, $\mathrm{p}<0.001$, aunque al hacer el análisis de acuerdo al tipo de institución agrupando los hospitales, no se encontraron diferencias significativas.

El estrés alto también fue más frecuente en los residentes (19\%) que en los médicos adscritos (11\%), p=0.006. Así mismo se encontraron niveles más altos de estrés en quienes trabajaban entre 70 y 86 horas por semana, $\mathrm{p}<0.001$; los que realizaban guardias $\mathrm{p}<0.001$; rotaban turnos $\mathrm{p}<0.001$ y no tenían descansos durante la jornada $\mathrm{p}<0.001$. (Cuadro III)

Así mismo, los mayores porcentajes de niveles de estrés alto y severo fueron reportados por quienes percibieron supervisión excesiva frecuentemente o siempre, $24.5 \%$ y $37.9 \%$ respectivamente, $\mathrm{p}<0.001$, mientras que el nivel alto registró altos porcentajes en aquellos que percibieron poca libertad para realización del trabajo $\mathrm{p}<0.001$; carga excesiva de trabajo $\mathrm{p}<0.001$ y número de pacientes vistos por día $\mathrm{p}<0.001$. (Cuadro III)

Cuadro III. Condiciones de trabajo y nivel de estrés en residentes y médicos de base de siete hospitales de la ciudad de México. México 2010

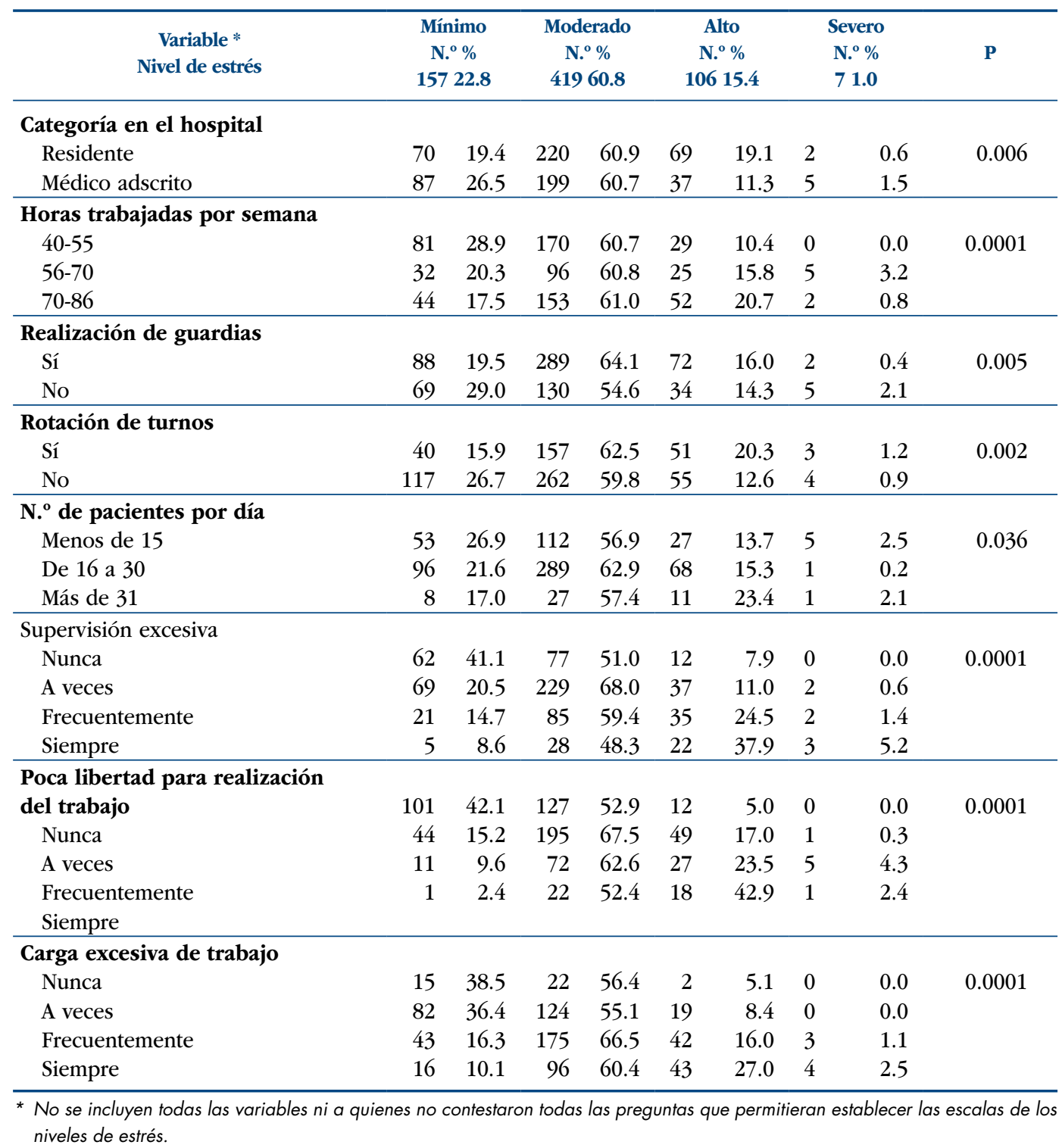




\section{Manifestaciones psicosomáticas (severidad de los síntomas)}

El instrumento para evaluar manifestaciones psicosomáticas mostró una alta consistencia interna de los elementos tipificados con un alfa de Cronbach de 0.83. La distribución de severidad de los síntomas mostró que la mayor frecuencia, $41 \%$, estuvo en el nivel leve; no obstante $25 \%$ se ubicó en un nivel alto y $10 \%$ en el severo. (Cuadro IV)

Las principales manifestaciones psicosomáticas referidas en la escala «mucha molestia" fueron: fatiga $29 \%$, dolor de espalda $22 \%$, dolor de cabeza $16 \%$, problemas para dormir $13 \%$, debilidad, dolor en articulaciones y en estómago, 11\%.

\section{Manifestaciones psicosomáticas, características socio-demográficas y condiciones de trabajo}

Las mujeres tuvieron mayor puntaje que los hombres en la escala de severidad de los síntomas, $\mathrm{p}<0.001$, ellas superaron el encontrado en el total de la población en los niveles alto y severo, con porcentajes de $34 \%$ y $16 \%$ respectivamente. Al igual que en los niveles de estrés, no encontramos diferencias según edad y estado civil.

Respecto a la prevalencia de las manifestaciones psicosomáticas en los médicos de los hospitales, los niveles más altos se observaron en el hospital de la SSA y uno del IMSS, $\mathrm{p}<0.001$.

En relación con la categoría de trabajo, los residentes en general tuvieron porcentajes más altos en las escalas leve y alta (46 y 26\%), mientras que los médicos adscritos la tuvieron en la severa (12\%). $\mathrm{p}=0.007$ (Cuadro IV)

No hubo diferencias significativas en la severidad de los síntomas de acuerdo con la antigüedad en el trabajo, tipo de servicio, horas trabajadas por semana, número de pacientes por día, ni número de descansos durante la jornada. Pero, si la hubo entre quienes realizaban guardias y los que no lo hacían, $\mathrm{p}<0.001$, el $30 \%$ de los primeros se ubicaron en el nivel alto. También se encontró que el 32\% de los que trabajaban en turno vespertino estuvieron en el grupo de severidad alta, de la misma forma, que el $29 \%$ de los que trabajaban con jornada acumulada, $\mathrm{p}=0.02$.

Así mismo y de manera semejante a lo ocurrido con los niveles de estrés, se encontró mayor severidad en los síntomas en los que refirieron supervisión excesiva, el porcentaje fue de $34 \%$, en el nivel alto cuando la supervisión era frecuente y de $27 \%$ en nivel severo cuando ésta era siempre. $\mathrm{p}<0.001$. (Cuadro IV)

Respecto a quienes refirieron poca libertad para realización de su trabajo de manera frecuente, se encontró que el $40 \%$ se ubicaba en el nivel alto de sintomatología, mientras que los que reportaron poca libertad siempre, estuvieron en la escala severa en un $29 \%$ $\mathrm{p}<0.001$. Lo mismo sucedió con carga excesiva de trabajo, en donde se encontró que el $34 \%$ tenía un nivel alto cuando ésta era frecuentemente y 19\% estaba en el nivel severo cuando era siempre. $\mathrm{p}<0.001$. (Cuadro IV)

Entre los que reportaron trabajar en otro lugar, además del lugar donde se realizó la encuesta, se encontró una diferencia significativa, $\mathrm{p}<0.001$, pero esta fue en sentido inverso de las anteriores, pues aquí hubo mayores porcentajes de niveles mínimo y leve en la severidad de los síntomas. 
Cuadro IV. Condiciones de trabajo y severidad de síntomas en residentes y médicos de base de siete hospitales de la ciudad de México. México 2010

\begin{tabular}{|c|c|c|c|c|c|c|c|c|c|}
\hline \multirow{2}{*}{$\begin{array}{c}\text { Variable * } \\
\text { Nivel de severidad }\end{array}$} & \multicolumn{2}{|c|}{$\begin{array}{l}\text { Mínimo } \\
\text { N. }{ }^{\circ} \% \\
\end{array}$} & \multicolumn{2}{|c|}{$\begin{array}{c}\text { Leve } \\
\text { N. }^{\circ} \% \\
29541.2\end{array}$} & \multicolumn{2}{|c|}{$\begin{array}{c}\text { Alto } \\
\text { N. } \% \\
18025.1\end{array}$} & \multicolumn{2}{|c|}{$\begin{array}{c}\text { Severo } \\
\text { N. }{ }^{\circ} \% \\
7310.2\end{array}$} & $\mathbf{P}$ \\
\hline & & & & & & & & & \\
\hline Residente & 72 & 19.1 & 172 & 45.7 & 99 & 26.3 & 33 & 8.8 & 0.007 \\
\hline Médico adscrito & 96 & 28.2 & 123 & 36.2 & 81 & 23.8 & 40 & 11.8 & \\
\hline \multicolumn{10}{|c|}{ Horas trabajadas por semana } \\
\hline $40-55$ & 80 & 27.6 & 117 & 40.3 & 62 & 21.4 & 31 & 10.7 & 0.301 \\
\hline $56-70$ & 35 & 21.6 & 70 & 43.2 & 43 & 26.5 & 14 & 8.6 & \\
\hline $70-86$ & 53 & 20.1 & 108 & 40.9 & 75 & 28.4 & 28 & 10.6 & \\
\hline \multicolumn{10}{|l|}{ Realización de guardias } \\
\hline Sí & 94 & 19.9 & 194 & 41.1 & 140 & 29.7 & 44 & 9.3 & 0.0001 \\
\hline No & 74 & 30.3 & 101 & 41.4 & 40 & 16.4 & 29 & 11.9 & \\
\hline \multicolumn{10}{|l|}{ Rotación de turnos } \\
\hline Sí & 47 & 18.3 & 94 & 36.6 & 87 & 33.9 & 29 & 11.3 & 0.0001 \\
\hline No & 121 & 26.4 & 201 & 43.8 & 93 & 20.3 & 44 & 9.6 & \\
\hline \multicolumn{10}{|l|}{ N. ${ }^{\circ}$ de pacientes por día } \\
\hline Menos de 15 & 57 & 27.7 & 92 & 44.7 & 41 & 19.9 & 16 & 7.8 & 0.196 \\
\hline De 16 a 30 & 101 & 21.9 & 184 & 39.8 & 124 & 26.8 & 53 & 11.5 & \\
\hline Más de 31 & 10 & 20.8 & 19 & 39.6 & 15 & 31.3 & 4 & 8.3 & \\
\hline \multicolumn{10}{|l|}{ Supervisión excesiva } \\
\hline Nunca & 57 & 36.3 & 62 & 39.5 & 26 & 16.6 & 12 & 7.6 & 0.0001 \\
\hline A veces & 84 & 23.9 & 150 & 42.7 & 89 & 25.4 & 28 & 8.0 & \\
\hline Frecuentemente & 18 & 12.0 & 63 & 42.0 & 51 & 34.0 & 18 & 12.0 & \\
\hline Siempre & 9 & 15.8 & 19 & 33.3 & 14 & 24.6 & 15 & 26.3 & \\
\hline \multicolumn{10}{|c|}{ Poca libertad para realización } \\
\hline $\begin{array}{c}\text { del trabajo } \\
\text { Nunca }\end{array}$ & 86 & 34.5 & 102 & 41.0 & 41 & 16.5 & 20 & $\begin{array}{l}8.0 \\
82\end{array}$ & 0.0001 \\
\hline Nunca & 65 & 21.4 & 133 & 43.8 & 81 & 26.6 & 25 & 8.2 & \\
\hline A veces & 12 & 10.3 & 42 & 35.9 & 47 & 40.2 & 16 & 13.7 & \\
\hline Frecuentemente & 5 & 11.9 & 17 & 40.5 & 8 & 19.0 & 12 & 28.6 & \\
\hline Siempre & & & & & & & & & \\
\hline \multicolumn{10}{|l|}{ Carga excesiva de trabajo } \\
\hline Nunca & 15 & 37.5 & 15 & 37.5 & 7 & 17.5 & 3 & 7.5 & 0.0001 \\
\hline A veces & 71 & 30.1 & 116 & 49.2 & 33 & 14.0 & 16 & 6.8 & \\
\hline Frecuentemente & 60 & 21.8 & 99 & 36.0 & 92 & 33.5 & 24 & 8.7 & \\
\hline Siempre & 21 & 13.1 & 63 & 39.4 & 46 & 28.8 & 30 & 18.8 & \\
\hline
\end{tabular}

* No se incluyen todas las variables estudiadas, ni a quienes no contestaron las preguntas que permitieran establecer las escalas de los niveles de severidad de síntomas.

\section{Evaluación del riesgo de estrés, severidad de síntomas y percepción de condiciones de trabajo entre médicos y residentes.}

Dado que en general se encontraron peores condiciones de trabajo, mayores niveles de estrés y severidad de síntomas en los residentes que en los médicos adscritos, para poder identificar diferencias en los riesgos de presentar estas alteraciones, se compactaron las escalas de clasificación. De esta forma para estrés, se establecieron dos nuevas categorías: sin estrés incluyendo a todos los que inicialmente estaban en los niveles de estrés mínimo o moderado; y con estrés, quienes habían pertenecido a los niveles de estrés alto y severo. Para severidad de los síntomas, se consideró nivel bajo a quienes se ubicaron en las modalidades mínimo y leve, y nivel alto a quienes se ubicaron en las categorías alto y severo. Lo mismo se hizo para las condiciones de trabajo percibidas, agrupando nunca o a veces, como no; y frecuentemente o siempre, como sí.

Se encontró que el riesgo de tener estrés en los residentes fue de 1.65 en comparación con los médicos adscritos, IC 95\% (1.12-2.43). Las tres condiciones de trabajo percibidas, 
en especial supervisión y carga de trabajo excesivas, estuvieron asociadas con un mayor riesgo para la presencia de estrés (Cuadro V)

En el caso de severidad de los síntomas psicosomáticos, prácticamente no hubo diferencias entre residentes y médicos adscritos, la razón de momios para la prevalencia fue de .979 (IC 95\% .920-1.33). Las condiciones de trabajo sí presentaron asociación con el nivel de severidad de los síntomas, aquí los más altos fueron carga excesiva y poca libertad para la realización del trabajo. (Cuadro V)

Cuadro V. Asociación entre categoría laboral, condiciones de trabajo percibidas, estrés y severidad de los síntomas psicosomáticos*, en médicos de siete hospitales de la ciudad de México. México, 2010

\begin{tabular}{lcccc}
\hline \multicolumn{1}{c}{ Variable } & $\begin{array}{c}\text { Estrés } \\
\text { Prevalencia } \\
\mathbf{x} 100\end{array}$ & $\begin{array}{c}\text { RMP } \\
\text { IC 95\% }\end{array}$ & $\begin{array}{c}\text { Severidad } \\
\text { de los síntomas } \\
\text { Prevalencia } \\
\mathbf{x} 100\end{array}$ & RMP IC95\% \\
\hline $\begin{array}{l}\text { Categoría laboral } \\
\quad \text { Residentes }\end{array}$ & 23.11 & 1.65 & 35.10 & .979 \\
$\quad$ Médicos adscritos & 15.38 & $1.12-2.43$ & 35.58 & $.720-1.33$ \\
\hline Supervisión excesiva & & & & \\
$\quad$ Sí & 36.04 & 4.01 & 47.78 & 2.084 \\
$\quad$ No & 12.32 & $2.69-5.96$ & 30.51 & $1.49-2.91$ \\
\hline Poca libertad para realización del trabajo & & & & \\
$\quad$ Sí & 35.71 & 3.35 & 53.20 & 2.62 \\
$\quad$ No & 8.52 & $2.22-5.05$ & 39.19 & $1.82-3.77$ \\
\hline Carga excesiva de trabajo & & & & \\
$\quad$ Sí & 25.83 & 3.46 & 44.31 & 2.91 \\
$\quad$ No & 9.12 & $2.16-5.57$ & 21.45 & $2.06-4.11$ \\
\hline
\end{tabular}

* Análisis de las categorías de respuesta compactadas para calcular el riesgo mediante la razón de momios para la prevalencia (RM).

\section{Asociación entre niveles de estrés y severidad de los síntomas}

Esta asociación se evaluó considerando las variables tanto de forma cualitativa como cuantitativa. En el primer caso, considerando los niveles compactados para analizar estrés-no estrés y su asociación con el nivel bajo y alto de severidad de los síntomas. Y en el segundo considerando la correlación de los puntajes obtenidos en dichas variables.

El riesgo de presentar nivel alto de severidad de los síntomas fue de 3.58 en quienes presentaban estrés, en comparación con quienes no lo presentaron, (IC 95 2.41-5.33) (Cuadro VI)

Cuadro VI. Asociación entre presencia de estrés* y severidad de los síntomas en médicos de siete hospitales de la ciudad de México. México 2010

\begin{tabular}{crcccc}
\hline \multirow{2}{*}{ Variable } & \multicolumn{3}{c}{ Severidad de los síntomas } & Prevalencia por 100 & RMP \\
\cline { 2 - 5 } & Alta & Baja & Total & & \\
\hline Estrés & & & & 60.0 & 3.589 \\
Sí & 78 & 52 & 130 & 29.47 & $2.41-5.33$ \\
No & 163 & 390 & 553 & 35.28 & \\
Total & 241 & 442 & 683 & & \\
\hline
\end{tabular}

* Análisis de las variables con categorías compactadas.

Se incluyen sólo los casos con respuestas completas para poder determinar las categorías.

Así mismo, el análisis de correlación mostró que en la población estudiada, a mayor presencia de estrés, los niveles de severidad de los síntomas fueron más altos. El coeficiente de correlación fue de $0.357, \mathrm{p}<0.001$. (Gráfico 1) 
Gráfico 1. Asociación entre severidad del estrés y severidad de los síntomas en médicos de siete hospitales de la ciudad de México, 2010

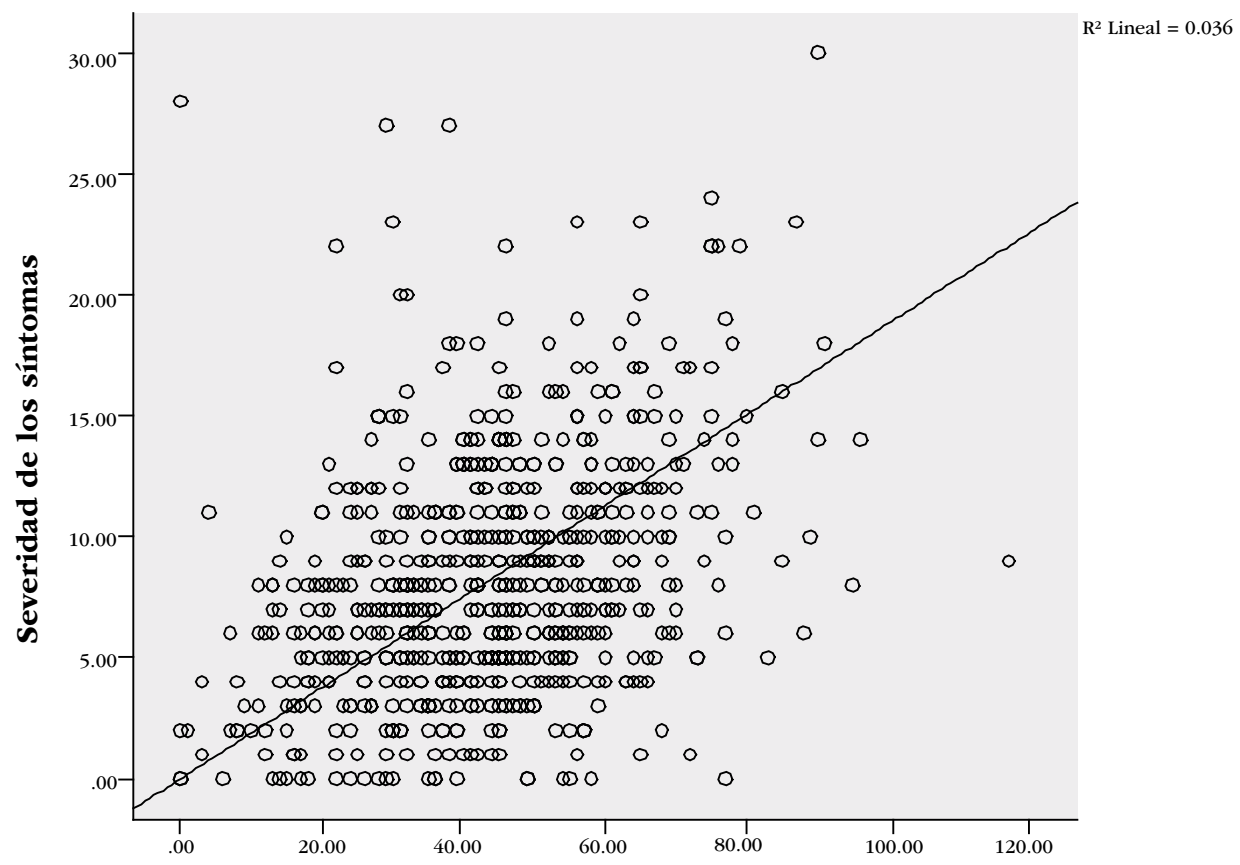

\section{DISCUSIÓN}

Los propósitos de este análisis fueron por una parte, revisar las características sociodemográficas y las condiciones de trabajo de médicos de siete hospitales de la ciudad de México y por la otra, evaluar la utilidad tanto del inventario de estrés en el trabajo médico de Wolfang ${ }^{12}$, como del cuestionario de severidad de síntomas de Kroenke ${ }^{13}$. Así mismo se planteó analizar las diferencias en la distribución de frecuencias de los niveles de estrés y síntomas según características personales y laborales.

Respecto a la selección de los hospitales y la población, es importante señalar que para su inclusión en este estudio, se tomaron en cuenta las características del sector salud en México, el cual no cuenta con un sistema universal de atención y seguridad social. Por ello y teniendo en cuenta las diferencias entre las instituciones que lo conforman, cuatro de los hospitales incluidos son parte del sistema de seguridad social, de estos, dos pertenecen al sistema de atención a los trabajadores al servicio del estado y dos al de de trabajadores de empresas de la iniciativa privada. Los otros tres forman parte de la Secretaría de Salud, cuyos médicos atienden a población abierta que no cuenta con seguridad social. Esto hace que los recursos de los que disponen estas instituciones sean distintos y por ello también las condiciones de trabajo. Las áreas de atención se seleccionaron considerando que existieran en todos los hospitales participantes.

Los resultados mostraron que las características socio-demográficas de la población fueron semejantes a las de otros reportes ${ }^{8,11}$.

Como ya ha sido mostrado en otras investigaciones ${ }^{7}$, los residentes fueron quienes principalmente percibieron exceso de supervisión, carga de trabajo excesiva, poca libertad para realizarlo, jornadas de trabajo extenuantes y gran número de pacientes atendidos por día. Mientras que entre los médicos adscritos hubo un alto porcentaje con doble empleo.

Respecto a la confiabilidad y utilidad de los instrumentos aplicados para la determinación de los niveles de estrés y severidad de los síntomas, se encontró una consistencia mayor a la reportada anteriormente para el inventario de Wolfang ${ }^{6} 0.91$ vs 0.83 y una exactamente igual en la aplicación del instrumento de Kroenke ${ }^{13}$, en donde el alfa fue igual a 0.83 . 
El promedio de estrés mostrado en este estudio fue mayor al detectado en otros que han utilizado el inventario de Wolfang ${ }^{6,12}$. La distribución en los diferentes niveles de estrés, no es comparable, ya que no se han reportado puntos de corte y los aquí definidos, se hicieron considerando la distribución de percentiles en esta población y las características de los puntos de corte establecidos en otras escalas. ${ }^{13,14}$ De acuerdo con los resultados, la mayoría de los médicos se ubicaron en el nivel moderado de estrés, pero una proporción importante (15\%) estuvo en el nivel alto. Esto se torna más relevante cuando se analizan las diferencias en estos niveles según las características personales y laborales de los médicos.

Al respecto, se encontraron diferencias significativas de acuerdo al hospital, los niveles más altos se ubicaron en dos de ellos con una alta demanda de atención, por lo que a futuro sería importante evaluar esta situación. Los porcentajes en los niveles alto y severo de estrés fueron mayores en los residentes.

Las características personales en general no mostraron asociación estadística, únicamente se observó un porcentaje ligeramente más alto en el estrés severo de los hombres.

De manera contraria, la gran mayoría de condiciones de trabajo percibidas por los médicos mostraron diferencias significativas. Aquí los porcentajes mayores de estrés alto y severo se encontraron en quienes percibieron frecuentemente o siempre supervisión, carga excesiva de trabajo y falta de libertad para realizarlo. Así como en los que tuvieron más horas de trabajo por semana, mayor número de pacientes y quienes tenían doble empleo.

Respecto a la severidad de los síntomas, la mayor parte de la población, se ubicó en el nivel moderado, no obstante, el nivel alto tuvo un porcentaje importante (25\%), mientras que el severo fue igual al reportado por Kroenke ${ }^{13}$.

La severidad de los síntomas fue mayor en las mujeres. Con el resto de características socio-demográficas, igual que en los niveles de estrés, no se encontraron diferencias.

Tampoco las hubo según la antigüedad en el trabajo, tipo de servicio, horas trabajadas por semana, doble empleo, ni número de pacientes por día. Pero sí, con responsabilidad excesiva, realización de guardias, turnos, carga de trabajo excesiva y, poca libertad para realizar el trabajo.

Por último, respecto a los límites y alcances del estudio, es necesario mencionar, que al tratarse de un diseño transversal, la información muestra únicamente lo que está sucediendo al momento de la entrevista y no sus causas, tampoco puede generalizarse a todos los médicos pues la muestra no es representativa. Así mismo, como es conocido existe posibilidad de un sub-registro de la información debido al síndrome del trabajador sano.

Entre los alcances, puede señalarse que es un estudio que aporta evidencia de la importancia de la asociación de las condiciones de trabajo médico con el estrés y las alteraciones en la salud. Así mismo, es de suma importancia la asociación encontrada entre los niveles de estrés y la severidad de los síntomas, pues aunque esto se ha hecho en otros estudios que han utilizado diversos instrumentos ${ }^{4,5,8}$, hasta el momento esta asociación no se había estudiado aplicando un inventario diseñado específicamente para medir el trabajo del personal de salud en México.

\section{CONCLUSIONES}

Los resultados de este estudio muestran características similares en las condiciones personales reportadas en otras investigaciones. Las condiciones de trabajo evidencian una carga excesiva, presión en el trabajo y jornadas extenuantes. Los instrumentos aplicados tuvieron una alta confiabilidad. El promedio y los niveles de estrés estuvieron 
por arriba de lo referido en las investigaciones de Wolfang y Román. Existen importantes diferencias en los niveles de estrés y severidad de síntomas de acuerdo con las condiciones de trabajo, a peores condiciones existe mayor porcentaje en los niveles alto y severo de estrés. Es necesario llevar a cabo otras investigaciones que permitan definir las escalas y los puntos de corte más convenientes para la delimitación del estrés en los profesionales de la salud.

\section{REFERENCIAS BIBLIOGRÁFICAS}

1. Organización Internacional del Trabajo/Organización Mundial de la Salud (2004). «Factores psicosociales en el trabajo: naturaleza, incidencia y prevención. Informe del comité mixto OIT/OMS sobre medicina del trabajo». OIT Ginebra.

2. Instituto Superiore per la Prevenzione e Sicurezza del Lavoro (ISPESL) (2003). "Strees e Burnout. Come riconoscere e prevenire il rischio". Centro Riserche di Monte Porzio Catone ISPESL Roma.

3. NIOSH (1999). "Stress at work». National Institute for Occupational Safety and Health (NIOSH). Publications 99-101. NIOSH USA.

4. Juárez A. Factores psicosociales relacionados con la salud mental en profesionales de servicios humanos en México. Ciencia \& Trabajo 2004; 14: 189-196.

5. Buddeberg-Fischer B, Klaghofer R, Stamm M, Siegrist J, Buddeberg C. Work stress and reduced health in young physicians: prospective evidence from Swiss residents. Int Arch Occup Environ Health 2008. Publicado en internet en febrero de 2008. http://www.springerlink.com.

6. Román HJ. Estrés y burnout en profesionales de la salud de los niveles primario y secundario de atención Instituto Nacional de Salud de los Trabajadores. Rev Cub Salud Pública 2003;29:103-110.

7. HarveyS, Courcy F, Petit A, Hudon J, Teed M, Loiselle O, Morin A. Organizational interventions and mental health in the workplace: A synthesis of international approaches. Studies and research projects report. Institute de recherche Robert Sauvé en santé et en securité du travail 2006. http://www.irsst.qc. ca, Montreal Canada.

8. Martínez-Lanz P, Medina-Mora ME, Rivera E. Adicciones, depresión y estrés en médicos residentes. Revista Fac Med UNAM 2005; 48 (5): 191-197.

9. Aranda C, Pando M, Velázquez I, Acosta B. Síndrome de burnout y factores psicosociales en el trabajo en estudiantes del posgrado del departamento de Salud Pública de la Universidad de Guadalajara. Rev Psiquiatría Fac Med Barna 2003;30(4):193-199.

10. Austria-Corrales F, Cruz-Valdés B, Herrera-Kiengelher Loredmy, Vazquez-Garcia JC, Salas-Hernandez J. Síndrome de burnout en médicos mexicanos en entrenamiento durante una contingencia sanitaria por virus de influenza A H1N1. Gaceta Médica de México 2011; 147: 97-103.

11. Toral-Villanueva R, Aguilar-Madrid G, Juárez-Pérez CA. Burnout and patient care in junior doctors in Mexico City. Occupational Medicine 2009; 59: 8-13.

12. Wolfgang AP. The health professions stress inventory. Psychol reports 1988b;(62):220-2.

13. Kroenke K, Spitzer RL, Williams JBW. The PHQ-15: Validity of a New Measure for Evaluating the Severity of Somatic Symptoms. Psychosomatic Medicine 2002; 64:258-266.

14. Maslach C, Jackson SE. The measurement of experienced burnout. J Occupational Behavior 1981; 2:99-103. 